\title{
Reducción de la procrastinación académica mediante la Terapia de Aceptación y Compromiso: un estudio piloto
}

\section{Reducing academic procrastination by acceptance and commitment therapy: A pilot study}

\author{
Almudena López-López, Lucía Toca Pérez, José Luis González Gutiérrez, \\ Borja Matías Pompa y Miriam Alonso Fernández \\ Universidad Rey Juan Carlos, España
}

\begin{abstract}
Resumen: El presente estudio tiene como objetivo la evaluación del impacto e implementación de un programa grupal piloto basado en la ACT para la reducción de la procrastinación académica en estudiantes universitarios, analizando así mismo, los cambios producidos en el autoconcepto académico y elementos motivacionales. Los estudiantes participantes fueron asignados aleatoriamente a un grupo experimental $(N=7)$, en el que se aplicó un programa de cinco sesiones, y un grupo control sin intervención $(N=15)$. Los instrumentos de medida aplicados fueron la Escala de Evaluación de la Procrastinación, el Cuestionario MAPE-3 para la evaluación de la motivación, y la escala de autoconcepto académico perteneciente al Cuestionario AF-5. Los resultados muestran cambios estadísticamente significativos en procrastinación académica, miedo al fracaso y autoconcepto académico. Aunque modesto, este estudio presenta datos a favor de la aplicación de la ACT como una posibilidad terapéutica en la reducción de la procrastinación en los estudiantes universitarios.

Palabras clave: procrastinación académica; terapia de aceptación y compromiso; autoconcepto académico; motivación académica; miedo al fracaso.

Abstract: The objective of this study is to evaluate the impact and implementation of a pilot program based on ACT for reducing academic procrastination in university students. It will also analyse the changes produced in the academic self-concept and motivational elements. Students were randomly assigned to an experimental group $(N=7)$ receiving a program of five sessions or to a control group without intervention $(N=15)$. The measurement ins-
\end{abstract}

Los autores firmantes de este artículo forman parte del Departamento de Medicina y Cirugía, Psicología, Medicina Preventiva y Salud Pública e Inmunología y Microbiología Médica. Facultad de Ciencias de la Salud, Universidad Rey Juan Carlos, Madrid, España.

La correspondencia sobre este artículo debe enviarse a la primera autora al email: almudena.lopez@urjc.es

(cc) BY-NC-ND Este es un artículo Open Access bajo la licencia CC BY-NC-ND. 
truments used were the Procrastination Assessment Scale, the MAPE-3 Questionnaire for evaluation of motivation, and the academic self-concept scale from the AF-5 Questionnaire. The results showed statistically significant changes for academic procrastination, fear of failure and academic self-concept. Although modest, this study presents data favourable to the application of ACT as an effective therapeutic option for the treatment of procrastination in university students.

Keywords: Acceptance and commitment therapy; academic procrastination, self-concept; academic motivation; psychoeducational program.

La procrastinación es definida como la tendencia a perder el tiempo, demorar y posponer de forma intencionada tareas o situaciones en lugar de afrontarlas. Esta conducta representa una forma de afrontamiento evitativo que provoca consecuencias negativas para la persona, como la disminución de su bienestar e incluso un incremento del malestar psicológico (Steel, 2007). Por otra parte, la procrastinación afecta, no solo a la ejecución de las tareas, sino también al nivel de autoeficacia del individuo. Este hecho introduce al individuo procrastinador en un círculo vicioso, puesto que la disminución de la autoeficacia aumenta las posibilidades de procrastinación (Garzón y Gil, 2017).

La procrastinación puede tener lugar en diferentes ámbitos de la vida, siendo el académico uno de los más estudiados, presentándose en entre el $30 \%$ y el $60 \%$ de los estudiantes. Este tipo de procrastinación está relacionada con el bajo rendimiento académico (Grunschel et al., 2016), siendo superior su poder de predicción al de otras más estudiadas tradicionalmente y difícilmente controlables tales como las variables demográficas y el rendimiento académico previo (Garzón y Gil, 2017).

Cuando las personas procrastinan entran en conflicto entre lo que deberían y lo que quieren hacer, bloqueándose en el dilema de acercamiento-evitación y anticipan la acción como no atractiva y la recompensa como demasiado alejada en el tiempo (Sánchez, 2010). Es por ello que los procrastinadores generalmente aplazan las tareas que perciben como aversivas y escogen aquellas que les parecen placenteras y que proporcionan recompensas a corto plazo (Sánchez, 2010; Specter y Ferrari, 2000). En este sentido, la procrastinación supone un estilo de afrontamiento de tipo evitativo, de modo que se posponen aquellas actividades que o bien se perciben como desagradables, o bien evidencian las limitaciones del estudiante, que, evitando la actividad, evita así un posible fracaso (Dómenech y Gómez, 2011; Rodríguez y Clariana, 2017). Según Specter y Ferrari (2000) los procrastinadores de tipo evitativo, muestran dificultades para enfrentarse a la amenaza y ansiedad que supone el comenzar tareas nuevas, y suelen basar su afrontamiento en la evitación de la novedad, el esfuerzo y los desafíos (Dómenech y Gómez, 2011; Rodríguez y Clariana, 2017). De este modo, evitan activamente el inicio de las tareas académicas hasta que ya apenas existe tiempo para poder realizar un trabajo óptimo (Ferrari y Pychyl, 2012). Por otra parte, la procrastinación está asociada a la aparición de problemas de ansiedad en los estudiantes, tanto la relativa al inicio de la tarea, como a las consecuencias de la propia demora y la mala distribución del tiempo (Marquina, Gómez, Salas, Santibañez y Rumiche, 2016).

Respecto a los trabajos que tratan de explicar la conducta de procrastinación académica, la mayor parte de autores consideran que ésta se explica fundamentalmente por variables motivacionales intrínsecas y por un fallo en las estrategias de regulación (Ferrari y Pychyl, 2007; Grunschel et al., 2016). Dentro de los factores más relevantes se encuentran las bajas expectativas de logro, el miedo al fracaso, un bajo autoconcepto académico, una tarea percibida como aversiva y sin consecuencias positivas a corto plazo, la falta de perseverancia, una baja orientación temporal hacia el futuro, la percepción de una supuesta reducción de estados aversivos y ansiosos gracias a la evitación y/o demora de la tarea, y ciertos rasgos de personalidad tales como impulsividad, perfeccionismo, inseguridad (Angarita, 2014; Ferrari y Díaz-Morales, 2006; Ferrari y Pychyl, 2012; Rodríguez y Clariana, 2015; Steel, 2007; 2010). Teniendo esto en cuenta, la evitación de sensaciones y estados emocionales que al estudiante le resultan aversivos parece jugar un papel fundamental en el mantenimiento de la conducta de procrastinación. Dicho de otro modo, es la evitación de la experiencia del evento privado (emoción, pensamiento), también llamada evitación experiencial, lo que mantiene a largo plazo la conducta de 
procrastinación. La evitación experiencial hace referencia a una actitud de rechazo a determinadas experiencias privadas consideradas como desagradables y a todas las acciones que la persona lleva a cabo para evitar estar en contacto con estas experiencias (Wilson y Luciano, 2002; Hayes et al., 2004).

Strunk et al. (2013) desarrollaron un modelo teórico de la procrastinación compuesto por dos dimensiones. La primera dimensión hace referencia a las conductas académicas relacionadas con el manejo del tiempo, y estaría formada por dos niveles: procrastinación y participación oportuna. La otra dimensión está compuesta por la base motivacional de la conducta académica, que estaría formada por dos categorías la motivación al logro y la motivación por evitación (citado en Garzón y Gil, 2017).

A lo largo de los últimos años, se ha tratado de determinar qué tipo de técnicas y estrategias pueden reducir la procrastinación académica. Diferentes autores coinciden en que la procrastinación académica puede ser entrenada, modificada y mejorada (Ferrari y Díaz-Morales, 2006; Grunschel et al., 2016; Garzón y Gil, 2017; Tuckman, 2002; Sánchez, 2010) utilizando programas que tengan en cuenta tanto aspectos conductuales, cognitivos, metacognitivos y motivacionales. Desde la intervención psicoeducativa se han llevado a cabo programas conductuales y cognitivo-conductuales centrados en el establecimiento de rutinas, calendarios, metas y objetivos diarios, así como exposición a los eventos que evita el estudiante cuando procrastina. En estos programas el mecanismo mediador de cambio es la modificación de la conducta a través la reducción de las creencias irracionales que alimentan la procrastinación, tales como el perfeccionismo o el miedo al fracaso, centrándose en la modificación cognitiva de las razones que el individuo tiene para procrastinar (Garzón y Gil, 2017; Grunschel et al., 2016; Ozer et al. 2013). Pese a que estos estudios han obtenido resultados positivos, los tamaños del efecto no se informan en la mayoría de los casos o bien son pequeños. Por otra parte, la mayoría de trabajos presentan importantes limitaciones metodológicas, tales como ausencia de medidas estandarizadas que pongan a prueba la intervención (Neenan, 2008) y ausencia de grupo control (Uzun et al., 2013), o consisten en estudios de caso único (Dryden, 2012; Karas y Spada, 2009). Entre los programas más interesantes, Uzun y su equipo (2013) realizaron un estudio piloto con diez estudiantes a los que se aplicó una intervención centrada en la identificación y modificación de distorsiones cognitivas y creencias irracionales asociadas al mantenimiento de la procrastinación. Los resultados indicaron una disminución significativa de los niveles de procrastinación, si bien la ausencia de grupo control supone una importante limitación metodológica. Otro trabajo reciente, realizado por Lukas y Berking (2018), obtiene también buenos resultados mediante la aplicación, a través de Smartphone, de técnicas basadas en la reducción de las distorsiones cognitivas relacionadas con las razones para procrastinar y en técnicas operantes, si bien el tamaño del efecto es pequeño.

Según Tuckman (2002) los programas que tratan de reducir la procrastinación académica tendrían que centrarse fundamentalmente en dos puntos: facilitar por parte del alumno el reconocimiento de la falta de funcionalidad e imprecisión de las razones que ellos mismos exponen para procrastinar, y aumentar la motivación intrínseca por la tarea. El primer objetivo es común a los programas ya existentes, el segundo de ellos no suele abordarse en los programas que se han puesto en marcha hasta la fecha.

En los últimos años, enmarcadas dentro de la llamada tercera generación de terapias cognitivo conductuales, se han desarrollado diversos tipos de terapias contextuales cuyo objetivo es la reducción de la evitación experiencial. Dentro de las terapias contextuales la Terapia de Aceptación y Compromiso (ACT) resulta la más completa (Barraca, 2007; Márquez, 2016) y sus principales características la hacen idónea para el tratamiento de la procrastinación (Luciano y Valdivia, 2006). Esta terapia no se centra en la modificación de los eventos privados, sino en la capacidad de aceptación de los mismos y, por tanto, en la desaparición de la necesidad de su evitación, de modo que el individuo esté listo para desarrollar una conducta comprometida con sus metas. (Barraca, 2007; Hayes et al., 2004; Luciano y Valdivia, 2006; Luciano et al., 2006; Wilson y Luciano, 2002). En este sentido, no sería necesaria la modificación de las creencias irracionales que mantienen la conducta de procrastinación, sino el entrenamiento en estrategias de aceptación de los eventos privados evitados, así como en la adquisición del compromiso conductual de acción, es decir, de ejecución de las tareas académicas que antes se evitaban.

Dentro de las técnicas aplicadas en la Terapia de Aceptación y Compromiso destacan el uso de metáforas y paradojas, los ejercicios experienciales y el entrenamiento en mindfulness (Barraca, 2007). Esta última técnica ha sido aplicada en nuestro país en contextos educativos con distintos objetivos, como incrementar el rendimiento académico, mejorar el autoconcepto y reducir la ansiedad (Franco et al., 2010; Mañas et al., 2011); me- 
jorar las medidas de crecimiento y autorrealización personal (Franco et al., 2011); incrementar el compromiso académico y disminuir el burnout académico y el cansancio emocional (De la Fuente et al., 2010). Respecto a su aplicación en el ámbito universitario, si bien no se conocen estudios cuyo objetivo sea la disminución de la procrastinación, sí existen estudios previos que avalan su aplicabilidad en estudiantes universitarios. Por ejemplo, el trabajo de Guerra, et al. (2018) mostró como el trabajo mediante ACT aumentó la autocompasión, la aceptación y la toma de perspectiva de los eventos problemáticos del estudiante, lo que favoreció la adaptación al aula y la reducción del fracaso académico en estudiantes universitarios en situación de vulnerabilidad. Otro estudio, también con estudiantes universitarios, mostró la efectividad del entrenamiento en valores mediante la Terapia de Aceptación y Compromiso para el aumento del rendimiento académico (Chase et al., 2013). Por otra parte, en un trabajo reciente, se muestra que el trabajo desde ACT resulta efectivo para tratar los trastornos de adaptación con ánimo depresivo en estudiantes universitarios de entre 18 y 25 años (Crespo y Vaca, 2019).

Sin embargo, en nuestro conocimiento, no se han realizado estudios que analicen el impacto de un programa basado en la Terapia de Aceptación y Compromiso en la procrastinación académica. El programa que aquí se presenta, a diferencia de los anteriores, se sirve fundamentalmente de la Terapia de Aceptación y Compromiso, utilizando metáforas, paradojas y ejercicios experienciales como herramientas principales, sirviéndose, además, de ejercicios de mindfulness para trabajar los componentes aceptación y contacto con el momento presente.

El objetivo del presente estudio es la evaluación del impacto e implementación de un programa piloto basado en la Terapia de Aceptación y Compromiso para la reducción de la procrastinación académica en estudiantes universitarios, analizando así mismo, los cambios producidos en el autoconcepto académico y el nivel de motivación extrínseca e intrínseca al estudio. Se esperaba que, tras el programa, los participantes del grupo de tratamiento disminuyesen sus niveles de procrastinación con respecto a un grupo control sin tratamiento, así como aumentasen sus niveles de motivación intrínseca. Por otra parte, no se esperaban cambios en las razones que los estudiantes manifiestan para procrastinar, dado que el tipo de intervención aplicada no centra el esfuerzo en la modificación de estas razones o creencias, sino en la aceptación de los eventos privados negativos cuya evitación llevan a la conducta de procrastinación.

\section{Método}

\section{Participantes}

La muestra final estuvo formada por 22 estudiantes universitarios de Grado y Máster de los que 7 formaron parte del grupo experimental y 15 del grupo control. Los participantes fueron reclutados a través anuncios en los diferentes campus de la universidad y envío de información por correo electrónico (ver procedimiento). Los criterios de inclusión fueron: Estar matriculado en estudios de grado o master en la URJC; ser mayor de 18 años y menor de 40; presentar dificultades relacionadas con la procrastinación; firmar el consentimiento informado. Los criterios de exclusión fueron: participar, en el momento del estudio, en otros protocolos de investigación o intervención que supusiesen incompatibilidad o sesgo (vg. otros programas de intervención psicoeducativa en la comunidad universitaria); presentar trastornos de personalidad u otros diagnósticos psiquiátricos que dificultasen el seguimiento del programa grupal; no tener un nivel de idioma castellano equivalente a un $\mathrm{C} 2$. Todos los participantes del grupo experimental eran mujeres, con edades comprendidas entre los 19 y los 25 años, siendo la media de 21.86 años y la desviación típica de 2.47 . El 71.4\% estaban realizando estudios de grado (dos estudiantes el grado en psicología, dos el grado en terapia ocupacional y uno el grado en química) y el $28.6 \%$ de máster (máster en psicología general sanitaria). Respecto al grupo control, el $31.3 \%$ eran hombres y el $68.8 \%$ mujeres. Sus edades oscilaban entre los 19 y los 30 años, siendo la media de 23.81 años y la desviación típica de 2.32. El 60\% realizaban estudios de grado (dos el grado en psicología, dos el grado en química, dos el grado en magisterio en educación primaria, uno el grado en terapia ocupacional, uno el grado en medicina) y el $40 \%$ de máster (dos el master de psicología general sanitaria, dos el master de formación del profesorado, uno el master de acceso a la abogacía y uno el master de investigación e innovación 
en contextos educativos). Tanto el grupo experimental como el grupo control estuvieron conformados en un $100 \%$ por alumnos cuya lengua nativa era el castellano.

\section{Variables e Instrumentos}

Nivel de procrastinación académica. Se evaluó mediante la primera parte de adaptación al castellano realizada por Garzón y Gil (2017) de la Prueba PASS- Procrastination Assessment Scale-Students (Solomon y Rothblum, 1984). Esta parte está formada por un total de 18 ítems. Doce de ellos evalúan la frecuencia de la procrastinación, y son utilizados para el cálculo de la puntuación final. En estos ítems alumno ha de valorar, en una escala tipo Likert de 5 puntos (desde nunca hasta siempre), hasta qué punto pospone o retarda el inicio de diversas actividades. Un ejemplo de ítem de esta escala sería: "¿hasta qué punto pospones estudiar para los exámenes?". Los seis ítems restantes indican el grado en el que los alumnos desean reducir la frecuencia de su comportamiento, y su uso es meramente informativo para el clínico. La escala posee una adecuada consistencia interna, con un alpha de cronbach de 0.79 .

Razones para procrastinar. Se evaluaron mediante la segunda parte de la mencionada adaptación de la prueba Prueba PASS (Solomon y Rothblum, 1984; adaptación de Garzón y Gil (2017). Formada por 25 ítems, explora las razones cognitivo-conductuales que llevan al estudiante a procrastinar. Esta segunda parte consta de cinco factores: búsqueda de excitación, falta de energía y autocontrol, perfeccionismo, ansiedad a la evaluación y poca asertividad y confianza. El alumno ha de reflejar, en una escala tipo Likert de 5 puntos, en qué medida la razón reflejada en el ítem es aplicable a su persona, siendo 1 nada aplicable y 5 totalmente aplicable. Algunos ejemplos de las razones posibles que se indican en los ítems de la escala son: "tenías ganas de sentir la emoción de hacer esta tarea en el último momento" (búsqueda de excitación), "no tenías bastante energía para empezar la tarea" (falta de energía y autocontrol), "te pusiste metas muy altas y te preocupaba no poder alcanzarlas" (perfeccionismo), "estabas preocupado de que al profesor no le gustará tu trabajo" (ansiedad a la evaluación) y "no tenías confianza en ti mismo para hacer un buen trabajo” (poca asertividad y confianza). El alfa de Cronbach es de 0.86 .

Motivación intrínseca. Como medida para evaluar la motivación intrínseca de los participantes, se utilizaron las subescalas correspondientes al factor "motivación por la tarea" del Cuestionario Mape-3 de Alonso Tapia, Montero y Huertas (2000). El cuestionario permite obtener una puntuación total correspondiente al factor motivación por la tarea, así como una por cada subescala que lo compone: motivación por aprender (21 ítems), disposición al esfuerzo (9 ítems,) y desinterés por el trabajo y rechazo del mismo (13 ítems; correlación negativa con el factor). Cada ítem se responde en una escala dicotómica en la que el alumno ha de señalar si está o no de acuerdo con la afirmación que se presenta. Algunos de los ítems que se presentan en esta escala son "para mí el mejor premio por mi trabajo es lo que disfruto cuando estoy haciéndolo y veo como progreso" (motivación por aprender), "estoy de acuerdo con quienes piensan que soy una persona que trabaja demasiado” (disposición al esfuerzo) y "es frecuente que empiece cosas que después no termino" (desinterés por el trabajo y rechazo del mismo). Los coeficientes alpha de Crombach indican una adecuada consistencia interna: motivación por aprender, 0.75 ; disposición al esfuerzo 0.74 ; desinterés por el trabajo y rechazo del mismo 0.78.

Miedo al fracaso. El miedo al fracaso, se evaluó utilizando la subescala homónima del cuestionario Mape-3 de (Alonso Tapia et al., 2000). Esta subescala está formada por 17 ítems de respuesta dicotómica según sea o no aplicable la afirmación al alumno evaluado. Un ejemplo de pregunta perteneciente a esta subescala sería "cuando tengo tareas difíciles pienso que no voy a ser capaz de hacerlas bien y me cuesta mucho concentrar$m e$ ”. Posee una elevada consistencia interna (alpha de Cronbach $=0.86$ ).

Autoconcepto académico. Para la evaluación del autoconcepto académico se utilizó la subescala del mismo nombre del Cuestionario AF-5- Autoconcepto Forma 5 de García y Musitu (2014). Esta subescala está formada por 6 ítems, en los que el alumno ha de reflejar su grado de acuerdo, en una escala del 0 al 10, con diversas afirmaciones relativas a su autoconcepto académico. Se obtiene una puntuación que oscila entre 0 y 60 ; mayores puntuaciones indican un autoconcepto más positivo. Ejemplos de ítems son "Soy un buen estudiante" o "mis profesores me estiman". Posee un alpha de Cronbach de 0.88 . 


\section{Procedimiento}

Tanto el programa aplicado en el grupo experimental como el procedimiento de investigación fueron aprobados por el Comité de Ética de la Investigación de la Universidad Rey Juan Carlos (URJC).

Los participantes fueron informados de la posibilidad de la realización del programa a través de anuncios en los diferentes campus de la universidad, así como información por correo electrónico a través de los Coordinadores de Grado y profesores responsables de diversas asignaturas de distintos Grados y Master impartidos en la Facultad de Ciencias de la Salud de la URJC. A todos los participantes se les explicó en qué consistía el programa, el objetivo principal del mismo (la reducción de la procrastinación) y su duración, así como la posibilidad de entrar directamente al programa o bien a una lista de espera. A todos los participantes de la lista de espera se les dio la oportunidad posterior de realizar el programa.

Tras el proceso de publicitación del programa, un total de 34 estudiantes manifestaron su deseo de su realización; todos ellos cumplieron los criterios de inclusión. Una vez firmado el consentimiento informado, los participantes fueron aleatorizados en los grupos experimental (17 estudiantes) y control (18 en lista de espera), mediante un proceso de aleatorización simple con ayuda de un software informático. Tras ello, se convocó a los participantes para la cumplimentación de las medidas pre-test, así como la recogida de datos sociodemográficos. De los 34 estudiantes, 4 del grupo experimental y 3 del control no realizaron la evaluación pre-test. Una vez realizada esta evaluación, se llevó a cabo la intervención, tal como se describe en el siguiente apartado. De los 13 estudiantes del grupo experimental que cumplimentaron la evaluación, 6 no acudieron al programa o acudieron a menos de 4 sesiones, quedando fuera de los análisis. Al finalizar el programa de intervención, todos los participantes fueron de nuevo convocados con el fin de obtener las medidas post-test. De los estudiantes del grupo control, uno no cumplimentó la evaluación post, quedando fuera de los análisis. De este modo, un total de 22 estudiantes configuraron la muestra final objeto de estudio. Tanto la evaluación pre-test como post-test fueron realizadas por un evaluador ciego a la condición experimental con amplia experiencia en evaluación psicológica.

\section{Programa de intervención}

El programa de intervención constó de cinco sesiones grupales con periodicidad semanal y de dos horas de duración. Con el fin de que el estudiante compatibilizase el programa con sus estudios universitarios, se realizaron dos grupos, uno en turno de mañana, con cinco estudiantes, y otro en turno de tarde con tres estudiantes. Todas las sesiones fueron llevadas a cabo por la misma psicóloga experta.

Para lograr el objetivo propuesto, la disminución de la procrastinación, el programa se centró, fundamentalmente, en la aplicación de técnicas orientadas al aumento de los procesos de aceptación, defusión, valores y acción comprometida. Para mejorar el trabajo con esos procesos, se intervino también en los procesos de contacto con el momento presente. Para el trabajo en aceptación, que se desarrolló, fundamentalmente, en las sesiones tres a cinco, se utilizaron fundamentalmente ejercicios experienciales, técnicas de fisicalización de la emoción y metáforas; trabajando sobre todo las emociones relacionadas con el miedo al fracaso, la pereza y la ansiedad. El proceso de defusión cognitiva se trabajó tanto a través de metáforas como a través de ejercicios de entrenamiento. Este trabajo se inició desde la segunda sesión. Su entrenamiento se consideró prioritario en el tiempo ya que un pensamiento defusionado facilita tanto los procesos de aceptación, como la atención a las contingencias presentes, lo que disminuye la probabilidad de que las cogniciones y "razones" del alumno relacionadas con la procrastinación impidan y boicoteen las conductas de estudio u otras conductas importantes para la vida académica. Por otra parte, al tomar distancia de sus pensamientos, éstos no provocan de modo inmediato emociones negativas que dificulten el rendimiento o potencien patrones autorreferentes negativos (Masuda et al., 2004).

Respecto al trabajo en valores, el concepto de valor se introdujo ya desde la segunda sesión, en el trabajo psicoeducativo y autorreflexivo con la matriz de flexibilidad psicológica. En la tercera sesión, se comenzaron a identificar metas, que en sesiones posteriores facilitaron en trabajo en valores, así como se introdujo 
la conexión entre los valores y la acción comprometida. Se trabajó también, en esta misma sesión, el papel de la fusión cognitiva y la evitación experiencial en el alejamiento de los mismos a través de la metáfora del autobús. La identificación plena de los valores, así como el trabajo más profundo con la acción comprometida en conexión con los valores se decidió dejar para las sesiones cuatro y cinco. Aunque el trabajo en valores es un eje fundamental en ACT, en el presente programa se comenzó trabajando por otros procesos, debido, por un lado, a la dificultad del concepto mismo de valor y su identificación para el alumno y, por otro, a la corta duración del programa, que obligaba a renunciar a algunos contenidos en pro de otros en las sesiones iniciales. Tal y como señalan Luciano y Valdivia (2006), actuar conforme a una dirección valiosa no es posible si uno no es capaz de distanciarse de los eventos privados y de aceptarlos sin tratar de modificarlos, por lo que se decidió dar prioridad, en las tres primeras sesiones, a aquellas técnicas que facilitaron la defusión y la aceptación El trabajo en valores fue, no obstante, un pilar fundamental del cambio, ya que se utilizó para explorar en profundidad las razones y motivaciones que llevan a implicarse en los estudios, de modo que éste cobre un sentido vital más allá del mero aprobado.

Por otra parte, aunque los ejes centrales del programa fueron la aceptación, la defusión cognitiva y el trabajo en valores, se consideró relevante trabajar, durante las primeras sesiones, el contacto con el momento presente, con el fin de conseguir una mayor profundización en los ejercicios y metáforas propuestas, así como facilitar otros procesos. Este proceso se trabajó a través de ejercicios de mindfulness, y ciertos contenidos psicoeducativos relacionados con el funcionamiento de la procrastinación, el miedo al fracaso o el concepto de mindfulness y sus beneficios. En la tabla 1 figura un esquema de los objetivos que se trabajaron en cada sesión y los ejercicios y técnicas utilizadas.

\section{Medidas de implementación}

Siguiendo los criterios de Lichtein et al.(1994), se tuvieron en cuenta tres aspectos de la evaluación de la implementación de los programas: transmisión, recepción y generalización. Durante el desarrollo del programa, al finalizar cada sesión, se pidió a los participantes que cumplimentaran un cuestionario de comprensión y utilidad de los contenidos de la sesión (transmisión y recepción). Por otro lado, al finalizar el programa se aplicó un cuestionario de satisfacción final con el objetivo de evaluar el ajuste del programa a las expectativas de los participantes, la percepción de adecuación de los contenidos y la utilidad percibida de la intervención, y el uso de las estrategias aprendidas en el contexto natural de su día a día (transmisión, recepción y generalización).

En todas las sesiones se realizó un registro de asistencias y otro de cumplimiento de las tareas para casa encomendadas a los participantes.

\section{Análisis de datos}

En primer lugar, se analizaron los estadísticos descriptivos para todas las variables del estudio. Dado que el tamaño muestral no permitía asegurar el cumplimiento de los supuestos de normalidad se realizaron pruebas no paramétricas con el fin de comprobar el impacto del programa. Con el fin de comprobar la homogeneidad en la distribución de las puntuaciones en las variables estudiadas entre los grupos control y experimental previamente a la aplicación del programa, se utilizó la prueba U de Mann-Whitney para muestras independientes, comparando las medidas pre-test de ambos grupos en todas las variables objeto de estudio.

Con el objetivo de analizar el cambio en los niveles de procrastinación, las razones para procrastinar, los factores de motivación por la tarea, el miedo al fracaso y el autoconcepto relacionado con la intervención, se realizaron dos tipos de pruebas. Primeramente, con el objetivo de analizar si se habían producido cambios significativos en el grupo experimental respecto al grupo control tras el programa de intervención, se realizó un análisis de las diferencias en las puntuaciones pos-test entre el grupo experimental y el grupo control mediante la prueba U de Mann-Whitney. De manera añadida, se verificó si se habían producido mejoras sig- 
Tabla 1. Objetivos y técnicas del programa

\section{Objetivos}

Sesión 1 Presentación.

Trabajar los conceptos de procrastinación y evitación experiencial.

Identificar situaciones en las que procrastinan y que función cumple la procrastinación.

Entender la aceptación como alternativa a la evitación.

\section{Técnicas}

Psicoeducación.

Ejercicios de reflexión grupales.

Análisis funcional individual.

Ejercicio análisis coste-beneficio.
Sesión 2 Diferenciar entre pensamiento, emoción y conducta. Distanciarse del contenido literal de los pensamientos. Diferenciar los pensamientos, emociones y sensaciones que tenemos de lo que somos.

Conseguir la aceptación de pensamientos, emociones y sensaciones sin juzgar.

Contacto con el momento presente.
Matriz de flexibilidad psicológica.

Análisis de autorregistros.

Ejercicio de creación de diferentes finales para un mismo pensamientoEjercicio del bolígrafo (mindfulness).

Mindfulness.

Body scan.
Sesión 3 Conseguir la aceptación psicológica tratando de evitar la fusión cognitiva.

Comienzo del trabajo en valores: meta, valor, fusión y evitación.

Contacto con el momento presente.
Metáfora del autobús.

Metáfora del túnel.

Ejercicio de fisicalización.

Ejercicio de observación del flujo de la experiencia.
Sesión 4 Trabajar el miedo al fracaso.

Clarificación de valores.

Aceptación psicológica.
Ejercicios de escritura sobre fracaso académico.

Ejercicio del funeral.

Ejercicios de reflexión grupal.

Metáfora aprender a ser un experto en manejar la bicicleta.
Sesión 5 Clarificación de valores.

Acción comprometida.

Cierre del programa.
Cuestionario de valores.

Matriz de flexibilidad psicológica: área académica.

nificativas entre las puntuaciones pre-test y pos-test tanto en el grupo experimental como en el grupo control. Para ello se realizaron pruebas $\mathrm{W}$ de Wilcoxon para muestras relacionadas.

Para el análisis de las medidas de implementación, se calcularon los índices de tendencia central media y moda para el análisis del cuestionario de satisfacción general, y la media para el resto de variables continuas. 


\section{Resultados}

\section{Caracterización de la muestra de estudio}

En primer lugar, con el fin de caracterizar la muestra, se analizaron los estadísticos descriptivos para todas las variables del estudio. En la tabla 2 se muestran las medias y desviaciones típicas pre-test y pos-test de los grupos experimental y control para las variables procrastinación académica, razones para procrastinar (búsqueda de excitación, falta de energía y autocontrol, perfeccionismo, ansiedad a la evaluación y poca asertividad y confianza), miedo al fracaso, motivación por la tarea, motivación por aprender, disposición al esfuerzo, desinterés por el trabajo y rechazo del mismo, y autoconcepto académico.

Tabla 2. Medias y desviaciones típicas de las variables objeto de estudio correspondientes a las medidas pre y post tratamiento, con prueba $W$ de Wilcoxon para el contraste de diferencias, en los grupos experimental y control

\begin{tabular}{|c|c|c|c|c|c|c|c|}
\hline \multirow[b]{2}{*}{ Variable } & \multirow[b]{2}{*}{ Rango } & \multicolumn{2}{|c|}{$\begin{array}{c}\text { Grupo Control } \\
M(D T)\end{array}$} & \multirow{2}{*}{$\begin{array}{c}\text { Prueba } \\
\text { de } \\
\text { Wilcoxon } \\
(W)\end{array}$} & \multicolumn{2}{|c|}{$\begin{array}{c}\text { Grupo Experimental } \\
M(D T)\end{array}$} & \multirow{2}{*}{$\begin{array}{c}\text { Prueba de } \\
\text { Wilcoxon } \\
(W)\end{array}$} \\
\hline & & Pre-test & Post-test & & Pre-test & Post-test & \\
\hline Procrastinación & $12-60$ & $37.40(7.13)$ & $37.33(6.85)$ & -.484 & 41.14 (7.99) & $31.43(2.88)$ & $-2.366 * *$ \\
\hline Búsqueda de excitación & $5-25$ & $7.73(3.69)$ & $9.47(4.63)$ & $-2.053 *$ & $7.57(2.82)$ & $6.71(1.60)$ & 2.00 \\
\hline $\begin{array}{l}\text { Falta de energía y autocon- } \\
\text { trol }\end{array}$ & $10-50$ & $26.87(5.45)$ & $27.80(7.72)$ & -.798 & $29.71(5.85)$ & $31.86(7.03)$ & 15.5 \\
\hline Perfeccionismo & $3-15$ & $8.40(2.90)$ & $7.60(2.93)$ & -1.364 & $8.57(1.72)$ & $7(2.31)$ & 5.5 \\
\hline Ansiedad a la evaluación & $2-10$ & $4.93(2.66)$ & $5.93(2.72)$ & -1.383 & $3.86(1.95)$ & $3.57(1.27)$ & 4.00 \\
\hline Poca asertividad confianza & $6-30$ & $15.40(4.45)$ & $14.20(4.35)$ & -1.588 & $16(4.55)$ & $16(3.27)$ & 5.00 \\
\hline Miedo al fracaso & $0-17$ & $7.87(4.22)$ & $8.47(5.44)$ & -.871 & $8.57(5.71)$ & $6(4.12)$ & $-1.892 *$ \\
\hline Motivación por la tarea & $0-43$ & $27.87(7.62)$ & $28.07(7.21)$ & -.983 & $28.43(4.65)$ & $29.71(5.53)$ & 14.00 \\
\hline Motivación por aprender & $0-21$ & $15.40(3.42)$ & $15(3.70)$ & -.554 & $15.57(1.62)$ & $16.86(2.48)$ & 21.5 \\
\hline Disposición al esfuerzo & $0-9$ & $3.80(2.51)$ & $4.47(2.03)$ & -1.628 & $4.29(2.14)$ & $4.43(2.44)$ & 12.00 \\
\hline Desinterés por el trabajo & $0-13$ & $4.33(2.90)$ & $4.40(2.97)$ & -.046 & $4.43(2.88)$ & $4.57(3.55)$ & 14.00 \\
\hline Autoconcepto académico & $0-60$ & $40.33(8.22)$ & $43.53(7.49)$ & $-2.847 * *$ & $38.86(9.97)$ & $43(9.57)$ & $-2.201 *$ \\
\hline
\end{tabular}

$* p<0.05 ; * *<0.01$ 
Análisis del cambio en los niveles de procrastinación, razones para procrastinar, factores de motivación por la tarea, miedo al fracaso y autoconcepto relacionado con la intervención

La prueba U de Mann-Whitney permitió comprobar la homogeneidad en la distribución de las variables objeto de estudio entre los grupos control y experimental, ya que no se encontraron diferencias significativas previas al programa para ninguna de las variables estudiadas entre los grupos.

Una vez contrastada dicha homogeneidad, se procedió a realizar un análisis de las diferencias en las puntuaciones en pos-test entre el grupo experimental y el grupo control mediante la prueba U de Mann-Whitney. Se obtuvieron diferencias estadísticamente significativas en la variable procrastinación académica $(U=21 ; p<$ 0.05). Como prueba del tamaño del efecto se utilizó la $r$ de Rosenthal, cuyo valor indica con tamaño del efecto medio $(r=-0.5)$.

De manera adicional, se verificó la presencia de mejoras significativas entre las puntuaciones pre-test y postest en ambos grupos mediante la prueba W de Wilcoxon (Tabla 2). En el grupo experimental se encontraron diferencias significativas en las variables procrastinación académica $(p<0.05)$, autoconcepto académico $(p<$ $0.05)$ y miedo al fracaso $(p=0.05)$. Para valorar la magnitud de las diferencias se utilizó la r de Rosenthal, que reportó un tamaño del efecto alto para la procrastinación académica $(r=-0.894)$ y autoconcepto académico $(r$ $=-0.832)$ y moderado en el caso de la variable miedo al fracaso $(r=-0.71)$.

En el grupo control, se hallaron diferencias significativas para las variables búsqueda de excitación $(p<0.05)$ y autoconcepto académico $(p<0$.05). La magnitud de la diferencia es moderada tanto para la búsqueda de excitación $(r=-0.530)$ como para el autoconcepto académico $(r=-0.735)$.

\section{Resultados de implementación}

La media total de comprensión de los contenidos en las cinco sesiones fue de 4.9 sobre 5 . Respecto al nivel de utilidad percibido de los contenidos, la media de las 5 sesiones fue de 4.8 sobre 5 . La media de satisfacción general con el programa fue de 4.91 sobre 5. Para un mayor detalle, en la tabla 3 se presentan la media y la moda correspondientes a cada uno de los ítems del Cuestionario de Satisfacción Final. Respecto al grado en que los participantes recomendarían el programa a otros estudiantes, se obtuvo una media de 9.29 sobre 10 .

Con respecto a la asistencia a las sesiones, de los 7 participantes, 4 asistieron a todas las sesiones y 3 de ellos a 4 de las 5 sesiones. Respecto a la realización de tareas para casa, 4 de los participantes realizaron las todas tareas encomendadas, y 3 de ellos realizaron 3 tareas de las 4 encomendadas.

\section{Discusión}

El objetivo principal de esta investigación fue la realización de un estudio piloto con el fin de evaluar el impacto de un programa basado en la Terapia de Aceptación y Compromiso en la procrastinación, la motivación y el autoconcepto académico de un grupo de estudiantes universitarios. Con este fin, los participantes fueron divididos en un grupo experimental y uno de control, presentando ambos grupos puntuaciones medias similares en las variables mencionadas antes del tratamiento.

Tradicionalmente, los programas más utilizados en los centros educativos han sido aquellos centrados en intervenciones teóricas en los que se ofrece a los estudiantes información sobre técnicas de estudio o gestión del tiempo (Cerezo, Núñez, Fernández, Suárez y Tuero, 2011; Vieira y Vidal, 2006). Por otra parte, las intervenciones centradas en la reducción de la procrastinación presentan, en general, limitaciones importantes. Si bien la Terapia de Aceptación y Compromiso ha sido utilizada para un gran número de problemas tanto médicos como psicológicos, mostrando su efectividad y eficacia (Hayes, 2004; Hayes y Strosalhl, 2004; Wilson y Luciano, 2002), hasta la fecha no se conocen estudios que la apliquen al campo educativo universitario con el fin de disminuir la procrastinación. Dado que esta terapia está centrada en la activación y aceptación psicológica como forma de proporcionar repertorios de conductas flexibles y amplios, trabajando igualmente la conciencia plena 
Tabla 3. Media y moda de las puntuaciones a los ítems del Cuestionario de Satisfacción Final

\begin{tabular}{|c|c|c|}
\hline Ítem & $\overline{\mathbf{X}}$ & $\mathbf{M}_{\mathbf{O}}$ \\
\hline Los contenidos de las sesiones se ajustaron a los objetivos del programa & 4.71 & 5 \\
\hline Los contenidos son adecuados a la duración del programa & 4.57 & 5 \\
\hline Los contenidos del programa le han sido de utilidad & 4.86 & 5 \\
\hline Los contenidos del programa le han sido comprensibles & 4.86 & 5 \\
\hline La metodología del programa se ha ajustado a sus expectativas & 4.29 & 4 \\
\hline El material proporcionado para la realización del programa ha sido adecuado & 4.71 & 5 \\
\hline La estructura del programa ha sido adecuada & 4.14 & 4 \\
\hline La organización de las sesiones ha sido correcta & 4.57 & 5 \\
\hline La duración del programa le ha parecido apropiada & 4.14 & 4 \\
\hline $\begin{array}{l}\text { En las sesiones se ha sentido cómodo/a para poder opinar, reflexionar y compartir sus ex- } \\
\text { periencias }\end{array}$ & 4.86 & 5 \\
\hline Las tareas para casa han sido explicadas previamente & 4.86 & 5 \\
\hline Las tareas para casa le han resultado útiles & 4.86 & 5 \\
\hline El responsable del programa ha resuelto sus dudas & 5 & 5 \\
\hline El responsable del programa propicia la participación del grupo & 5 & 5 \\
\hline El programa le ha sido de utilidad para su vida académica & 4.71 & 5 \\
\hline
\end{tabular}

y los valores (Luciano y Valdivia, 2006; Hayes, 2004; Ribero y Agudelo, 2016) resulta muy adecuada, además de para el tratamiento de patologías, para tratar problemas cotidianos en población no clínica.

Tras la realización del presente programa, el nivel de procrastinación y el miedo al fracaso de los participantes del grupo experimental disminuyó significativamente. Los participantes del grupo experimental obtuvieron una reducción estadísticamente significativa de la procrastinación en comparación con el grupo control, donde los niveles no se modificaron. Esta reducción en los niveles de procrastinación se ha conseguido previamente a través de técnicas cognitivo-conductuales dirigidas a la modificación de las creencias ligadas a las razones para procrastinar (Aljoscha y Berking, 2018; Uzun et al., 2013). A diferencia de estos programas, en la presente 
intervención, la reducción en la procrastinación no vino acompañada de este cambio en las razones. A lo largo del programa se pudieron comprobar las razones para procrastinar que manifestaban los participantes del programa. Gran parte de ellas estaban relacionadas, tal y como señalan Tuckman (2002), Grunschel et al. (2016) y Garzón y Gil (2017), con el miedo al fracaso, una baja percepción de autoeficacia, una falta de motivación hacia las tareas y el perfeccionismo. El hecho de que se haya producido una disminución de la procrastinación sin necesidad de un cambio en las razones para procrastinar, es coherente con el tipo de intervención realizada. ACT no se basa en la modificación del contenido de los pensamientos automáticos y las creencias, sino en el entrenamiento en estrategias de defusión y aceptación, que permitirán al alumno realizar una acción comprometida con los valores, en este caso aquellos valores relacionados con las metas de estudio. De este modo, no es necesaria una modificación del contenido de las razones para procrastinar, sino que basta con que el alumno no se fusione con tales razones, es decir, no deje que tales razones y los pensamientos automáticos derivados de ellas actúen como reglas que guíen su conducta. A pesar de que no se trabajó en técnicas de reestructuración cognitiva, los participantes, en el transcurso del programa, pudieron ser capaces de admitir y ser conscientes de la irracionalidad de sus razones para procrastinar, si bien, dada la naturaleza de la intervención, no se trató de reestructurar tales cogniciones. Esta toma de conciencia sobre la escasa correspondencia entre la realidad y las creencias, se pudo constatar en las respuestas que daban a los ejercicios experienciales, en los comentarios de las metáforas, además de en los cambios que se produjeron entre la matriz de flexibilidad psicológica realizada en la primera sesión y la realizada en la última, cuyos datos no se presentan en este artículo dado su carácter cualitativo y su extensión. El paso previo de tomar conciencia de que tales razones son contenidos verbales que, en muchas ocasiones, pueden no corresponder a la realidad objetiva, ayuda en el proceso de defusión, sin ser necesario sustituir estas creencias por otras más "racionales". Este proceso de defusión, que fue entrenado, además, con técnicas específicas, facilita el procesamiento de las contingencias presentes y los procesos conductuales de acción comprometida, contrarios a la procrastinación. Por otra parte, como se ha comentado en el apartado de metodología, parte de las técnicas aplicadas se orientaron al trabajo con los valores del alumno relacionados con el mundo académico. El trabajo en valores permitió al alumno explorar las razones y motivaciones que le llevan a implicarse en los estudios que está cursando, de modo que la acción de estudiar cobra un sentido vital más allá del mero aprobado.

Aunque la capacidad de atención y concentración no se ha evaluado como variable dependiente, es probable que el entrenamiento en defusión mejore también, a medio y largo plazo el rendimiento no sólo por la vía de disminuir la procrastinación, sino mejorando la capacidad de atención y concentración del estudiante, dado que la defusión disminuye los bucles de cognición perseverativa, tales como la rumiación y la preocupación (Gillanders et al., 2014), que a su vez disminuyen la capacidad de concentración en las tareas académicas (Lyubomirsky et al., 2003).

Respecto al miedo al fracaso, variable contemplada dentro de las medidas motivacionales, los análisis prepost realizados para el grupo experimental indican que los participantes en el programa redujeron significativamente el miedo al fracaso. Aunque las diferencias post evaluación no reflejan una distancia significativa con respecto al grupo control, el hecho de que los participantes del grupo control no modificasen sus puntuaciones de miedo al fracaso parece apuntar a que la reducción experimentada por los asistentes al programa está relacionada con las variables modificadas a través del mismo. Pese a que estudios previos señalan esta variable como un posible mediador del cambio, ninguno de estos trabajos reporta datos cuantitativos sobre la reducción del miedo al fracaso tras el tratamiento cognitivo-conductual (Garzón y Gil, 2017; Grunschel et al., 2016; Ozer et al. 2013). Los datos del presente trabajo parecen apoyar la aplicación de ACT para la disminución de esta variable, si bien el tamaño muestral no permitió realizar análisis adecuados para contrastar su papel como mediador del cambio en la procrastinación.

En cuanto al autoconcepto académico, éste aumenta tras la aplicación del programa, si bien este aumento no es superior al experimentado por el grupo control, cuyos participantes presentan también un mejor autoconcepto en el momento post evaluación. Este hecho parece indicar que, probablemente, las causas de dicha mejora sean externas al programa.

Con respecto a las medidas de implementación, en general los estudiantes que participaron en el programa valoraron éste muy positivamente. Especialmente valoraron positivamente la utilidad de los contenidos para 
su vida personal y académica, el ajuste de las sesiones a los objetivos del programa, la resolución de dudas que se hizo durante las sesiones, el hecho de que durante el programa se fomentara la participación de todos los componentes del grupo, así como el material proporcionado en las sesiones y la utilidad de las tareas para casa. Por otro lado, el nivel de comprensión, utilidad y satisfacción con las sesiones fue en general alto destacando las valoraciones referentes a la segunda y cuarta sesión, donde la satisfacción y la utilidad de los contenidos obtuvieron la puntuación máxima.

A través de los comentarios que los participantes realizaron en el Cuestionario de Satisfacción Final, se comprobó que los estudiantes, en general, valoran muy positivamente todas aquellas intervenciones y programas que se llevan a cabo en los contextos educativos para tratar de mejorar sus habilidades académicas y su rendimiento académico. Además, consideraron positiva la metodología participativa y el poder realizar este tipo de programas de manera grupal, junto con otros miembros de la comunidad universitaria. Gran parte de los participantes expresaron que les hubiera gustado que el programa tuviera mayor número de sesiones y que incorporara otro tipo de técnicas y herramientas, fundamentalmente de relajación, organización y planificación.

La presente investigación presenta varias limitaciones que es importante tener en cuenta. En primer lugar, debido a que se trataba de un estudio piloto, el tamaño muestral es muy reducido, lo que limita la generabilidad de los resultados. Segundo, existe un desequilibrio entre el número de participantes del grupo control y el número de participantes del grupo experimental. Además, todos los participantes del grupo experimental fueron mujeres. Tercero, casi todas las participantes del grupo experimental realizaban estudios relacionados con las ciencias de la salud, en concreto psicología y terapia ocupacional. Es por ello que no podemos asegurar que el programa tenga el mismo impacto en estudiantes de otras ramas de conocimiento. Cuarto, no se trabajó con una muestra aleatoria, sino que estaba formada por estudiantes que se prestaron voluntarios, por lo que pueden existir sesgos. En quinto lugar, no se ha realizado un seguimiento en el tiempo de los resultados. Sexto, el número de sesiones es relativamente corto, lo que no permite trabajar en profundidad todos los contenidos. Se optó por un número reducido de sesiones para aumentar las posibilidades de adherencia, ya que actividades de mayor duración son difíciles de completar por el alumnado, dada la carga de trabajo. Aun así, hubo un número significativo de estudiantes que no participa del total de las sesiones, lo que supone una limitación añadida. Posiblemente un programa más extenso, con un mayor número de sesiones hubiera permitido observar mayores modificaciones en la procrastinación, motivación y autoconcepto académico de los participantes. En relación a esto, algunos estudiantes manifestaron su deseo de que el programa hubiese tenido un mayor número de sesiones. Por otra parte, si bien se comenzó a trabajar la identificación de metas y se introdujo el concepto de valor desde la segunda sesión, el trabajo profundo con los valores y la acción comprometida no se realizó hasta la cuarta sesión. Puede que un trabajo en profundidad de los valores desde las primeras sesiones, o bien un aumento del número de sesiones con el fin de aumentar el tiempo dedicado a estos procesos, hubiesen permitido un cambio mayor en las variables objeto de estudio.

Teniendo en cuenta estas limitaciones, un aspecto de interés de cara a futuras investigaciones sería realizar un seguimiento a tres y seis meses del programa para poder comprobar si los cambios producidos por el programa se mantienen con el tiempo. Ribero y Agudelo (2016) en una intervención en dos casos de evitación experiencial basada en la terapia de aceptación y compromiso realizaron una fase de seguimiento a los tres meses, además de dos sesiones de seguimiento una tras una semana de la finalización de la intervención y otras a los quince días para conseguir un mayor control. Igualmente, sería de interés realizar el programa en una muestra más amplia, con estudiantes de diferentes áreas académicas y con un mayor número de sesiones en el que puedan trabajarse en mayor profundidad todos los elementos de la terapia de aceptación y compromiso y combinarlo con sesiones de psicoeducación más amplias.

En conclusión, aunque modesto y con las limitaciones señaladas, este estudio presenta datos a favor de la aplicación de la ACT como una posibilidad terapéutica eficaz en el tratamiento de problemas cotidianos en los estudiantes universitarios, como la procrastinación y el miedo al fracaso. Futuras investigaciones han de contrastar el impacto de un programa de mayor extensión, incluso que combine las técnicas propias de ACT con otras técnicas psicoeducativas como la gestión del tiempo y la relajación, así como su impacto en otras variables dependientes, como la concentración en la tarea y el rendimiento académico. 


\section{Referencias}

Alonso, J., Montero, I. y Huertas, J. A. (2000). Evaluación de la motivación en sujetos adultos: el cuestionario MAPE-3. Facultad de Psicología: Universidad Autónoma de Madrid. http://sohs.pbs.uam.es/webjesus/ eval psicologica/cuestionarios/mape3.pdf

Angarita, D. (2014). Algunas relaciones entre la procrastinación y los procesos básicos de motivación y memoria. Revista Iberoamericana de Psicología: Ciencia y Tecnología, 7(1), 91-101.

Barraca, J. (2007). La terapia de aceptación y compromiso (ACT). Fundamentos, aplicación en el contexto clínico y áreas de desarrollo. Miscelanea Comillas, 127(65), 761-781.

Cerezo, R., Núñez, J. C., Fernández, E., Suárez, N; Tuero, E. (2011). Programas de intervención para la mejora de las competencias de aprendizaje autorregulado en educación superior. Perspectiva Educacional, 50(1), 1-30.

Chase, J., Houmanfar, R., Hayes, S., Ward, T., Plumb., J. y Follette, V. (2013). Values are not just goals: Online ACT-based values training adds to goal setting in improving under graduate college student perfomance. Jorunal of Contextual Behavioral Science, 2(3-4), 79-84.

Crespo, M. C. y Vaca, E. N. (2019). Terapia de aceptación y compromiso para el trastorno de adaptación con ánimo depresivo en estudiantes universitarios entre 18 y 25 años. [Tesis, Universidad San Francisco de Quito, USFQ]. Repositorio Digital USFQ. http://repositorio.usfq.edu.ec/handle/23000/8112

De la Fuente, J., Franco, C. y Mañas, I. (2010). Efectos de un programa de entrenamiento en conciencia plena (mindfulness) en el estado emocional de estudiantes universitarios. Estudios sobre educación, 19, 31-52.

Dómenech, F. y Gómez, A. (2011). The relationship among student basic need satisfaction, approaches to learning, reportiong of avoidance strategies and achievement, Electronic Journal of Research in Educational Psychology, 9(2), 463-496.

Dryden, W. (2012). Dealing with procrastination: the REBT approach and a demonstration session. Journal of Rational-Emotive \& Cognitive-Behavior Therapy, 30(4), 264-281.

Ferrari, J. R. y Díaz-Morales, J. F. (2006). Procrastination: different time orientations reflect different motives. Journal of research in Personality, 41(3), 707-714.

Ferrari, J.R. y Pychyl, T.A. (2007). Regulating speed, accuracy, and judgments by indecisives: Effects of frequent choices on self-regulation failure. Personality and Individual Differences, 42(4), 777-787. https://doi.org/10.1016/j.paid.2006.09.001

Ferrari, J. R. y Pychyl, T. A. (2012). "If I wait, my partner will do it": the role of conscientiousness as a mediator in the relation of academic procrastination and perceived social loafing. North American Journal of Psychology, 14(1), 13-24.

Franco, C., De la Fuente, M. y Salvador, M. (2011). Impacto de un programa de entrenamiento en conciencia plena (mindfulness) en las medidas del crecimiento y la autorrealización personal. Psicothema, 23(1), 58-65.

Franco, C., Soriano, E. y Justo, E. (2010). Incidencia de un programa psicoeducativo de mindfulness (conciencia plena) sobre el autoconcepto y el rendimiento académico de estudiantes inmigrantes sudamericanos residentes en España. Revista Iberoamericana de Educación, 53(6), 1-13.

García, F. y Musitu, G. (2014). AF5: Autoconcepto Forma 5, (4ª Ed.). TEA Ediciones.

Garzón, A. y Gil, J. (2017). Propiedades psicométricas de la versión en español de la prueba PASS. Revista Iberoamericana de Diagnóstico y Evaluación Psicológica, 1(43), 149-163.

Gillanders, D., Bolderston, H., Bond, F., Dempster, M., Flaxman, P., Campbell, L., Kerr, S., Tansey, L.,Noel, P., Ferenbach, C., Masley, S. Roach, L., Lloyd, J., May, L. Clarke, S. y Remington, B. (2014). The Development and Initial Validation of the Cognitive Fusion Questionnaire. Behavior Therapy, 45(1), 83-101. https:// doi.org/10.1016/j.beth.2013.09.001

Grunschel, C., Schwinger, M., Steinmayr, R. y Fries, S. (2016). Effects of using motivational regulation strategies on students academic procrastination, academic performance, and well-being. Learning and Individual Diferences, 49, 162-170. https://doi.org/10.1016/j.lindif.2016.06.008

Guerra, S. P., Pérez, L. B. y Trujillo, L. F. (2018). Efectividad de un programa de autocompasión basado en ACT en un contexto de educación inclusiva. [Trabajo fin de Grado, Corporación Universitaria Minuto de Dios]. Sistema Nacional de Bibliotecas Rafael García-Herreros. http://hdl.handle.net/10656/6967 
Hayes, S. C. (2004). Acceptance and Commitment Therapy and the new behavior therapies: Mindfulness, acceptance and relationship. En S. C. Hayes, V. M. Follette y M. M., Linehan (Eds.), Mindfulness and acceptance: Expanding the cognitive behavioral tradition (pp. 1-29). Guilford.

Hayes, S. C; Strosahl, K. D. y Wilson, K. G. (2004). Terapia de Aceptación y Compromiso: proceso y práctica del cambio consciente (Mindfulness). Desclée de Brouwer.

Karas, D., Spada, M.M., 2009. Brief cognitive-behavioural coaching for procrastination: a case series. Coaching 2(1), 44-53.

Luciano, M. C. y Valdivia, M. S. (2006). La terapia de aceptación y compromiso (Act): fundamentos, características y evidencia. Papeles del psicólogo, 27(2), 79-91.

Luciano, M. C., Valdivia, M. S., Gutiérrez, O. y Páez, M. (2006). Avances desde la terapia de aceptación y compromiso (ACT). EduPsykhe, 5(2), 173-201.

Lichtein, K. L., Riedel, B. W. y Grieve, R. (1994). Fair tests of clinical trials: A treatment

implementation model. Advances in Behaviour Research and Therapy, 16(1), 1-29.

Lukas, C. A. y Berking, M. (2018). Reducing procrastination using a smartphone-based treatment program: A randomized controlled pilot study. Internet Interventions, 12, 83-90. https://doi.org/10.1016/j.invent.2017.07.002

Lyubomirsky, S., Kasri, F. y Zehm, K. (2003). Dysphoric Rumination Impairs Concentration on Academic Tasks, Cognitive Therapy and Research, 27,309-330. https://doi.org/10.1023/A:1023918517378

Mañas, I., Franco, C., Cangas, A. y Gallego, G. (2011). Incremento del rendimiento académico, mejora del autoconcepto y reducción de la ansiedad en estudiantes de bachillerato a través de un programa de entrenamiento en mindfulness (conciencia plena). Encuentros en psicología, 28, 44-62.

Márquez, M. (2016). Tendencias actuales en intervención psicológica. Madrid: Síntesis.

Marquina, R. J., Gómez, L., Salas, C., Santibañez, S. y Rumiche, R. (2016). Procrastinación en alumnos universitarios de Lima Metropolitana. Revista Peruana de Obstetricia y Enfemería, 12(1). https://www.aulavirtualusmp.pe/ojs/index.php/rpoe/article/view/747/585

Masuda, A. Hayes, S., Sackett, C. y Twohig, M. (2004). Cognitive defusion and self-relevant negative thoughts: examining the impact of a ninety year old technique. Behaviour Research and Therapy, 42(4) 477-485.

Neenan, M. (2008). Tackling procrastination: An REBT perspective for coaches. Journal of Rational-Emotive \& Cognitive-Behavior Therapy, 26(1), 53-62. https://doi.org/10.1007/s10942-007-0074-1

Ozer, B. U., Demir, A. y Ferrari, J. (2013). Reducing academic Procrastination through a group greatment grogram: A pilot study. Journal of Rational-Emotive \& Cognitive-Behavior Therapy, 31, 127-135. https:// doi.org/10.1007/s10942-013-0165-0

Pychyl, T. A., Lee, J. M., Thibodeau, R. y Blunt, A. (2000). Five Days of Emotion: An Experience Sampling Study of Undergraduate Student Procrastination. Journal of Social Behavior and Personality, 15(5), 239-254.

Ribero, S. y Agudelo, L. (2016). La aplicación de la terapia de aceptación y compromiso en dos casos de evitación experiencial. Avances en Psicología Latinoamericana, 34(1), 29-46. https://doi.org/10.12804/ ap134.1.2016.03

Rodríguez, A. y Clariana, M. (2017). Procrastinación en estudiantes universitarios: su relación con la edad y el curso académico. Revista Colombiana de Psicología, 26(1), 45-60. https://doi.org/ 10.15446/rcp. v26n1.53572

Sánchez,A. M. (2010). Procrastinación académica: un problema en la vida universitaria. Studiositas, 5(2), 87-94.

Solomon, L. J. y Rothblum, E. D. (1984). Academic procrastination: Frequency and cognitive-behavioral correlates. Journal of Counseling Psychology, 31(4), 503-509. https://doi.org/10.1037/0022-0167.31.4.503

Specter, M. H. y Ferrari, J. R. (2000). Time orientations of procrastinators: Focusing on the past, present, or future? Journal of Social Behavior and Personality, 15(5), 197-202.

Steel, P. (2007). The nature of Procrastination: A Meta-analytic and theoretical review of quintessential self-regulatory failure. Psychological Bulletin, 133(1), 65-94. https://doi.org/10.1037/0033-2909.133.1.65

Strunk, K., Cho, Y., Steele, M. y Bridges, S. (2013) Development and validation of a 2 x 2 model of time-related academic behavior: procrastination and timely engagement. Learning and individual differences, 25, 35-44. https://doi.org/10.1016/j.lindif.2013.02.007 
Tuckman, B. W. (2002, 22-25 Agosto). Academic procrastinator: their rationalizations and web-course performance [Symposium Paper]. Annual Meeting of the American Psychological Association, Chicago. https:// files.eric.ed.gov/fulltext/ED470567.pdf

Vieira, M. J. y Vidal, J. (2006). Tendencias para la educación superior europea e implicaciones para la orientación universitaria. Revista Española de Orientación y Psicopedagogía, 17(1), 75-97.

Wilson, K. G. y Luciano, M. C. (2002). Terapia de Aceptación y Compromiso (ACT): un tratamiento conductual orientado a los valores. Ediciones Pirámide.

Artículo recibido: 04/06/2019

Artículo aceptado: 12/02/2020 